\title{
EVALUATION OF FALSE ALARM RATES OF A WALKTHROUGH DETECTION PORTAL DESIGNED FOR DETECTING TRIACETONE TRIPEROXIDE (TATP) VAPOUR FROM FIELD TEST RESULTS AND RECEIVER OPERATING CHARACTERISTIC (ROC) CURVES
}

\author{
Y. TAKADA ${ }^{1}$, H. NAGANO ${ }^{1}$, Y. KAWAGUCHI ${ }^{1}$, Y. SUZUKI ${ }^{1}$, E. NAKAJIMA ${ }^{2}$, \\ M. SUGIYAMA ${ }^{1}$, M. SUGAYA ${ }^{1}$, Y. HASHIMOTO ${ }^{1} \&$ M. SAKAIRI ${ }^{1}$ \\ ${ }^{1}$ Central Research Laboratory, Hitachi, Ltd, Japan. \\ ${ }^{2}$ Tachikawa Office, WDB Co, Ltd, Japan.
}

\begin{abstract}
We have been developing a "walkthrough detection portal system" with the aim of preventing terrorist attacks or criminal bombings in crowded public places. The portal system consists of a vapour sampler, an atmospheric-pressure chemical ionisation (APCI) ion source, and an explosives detector based on ion-trap mass spectrometry (ITMS). The system can detect triacetone triperoxide (TATP) vapour at a high throughput (1,200 persons per hour). We tested the two-portal systems at the automated ticket gate areas of a train station to obtain background signal data from passengers. The field test results for 9,951 passengers indicated that the false positive rate of the portal system for TATP detection was below $0.01 \%$ when multi-marker detection logic was used, although it was about $0.5-2.0 \%$ with single marker detection $(\mathrm{m} / \mathrm{z}, 75,77$ or 91$)$. We used the receiver operating characteristic (ROC) curves obtained from the field test results at the train station and the calibration curves of the walkthrough detection portal system to evaluate the relationship between a true positive rate and a false positive rate. The ROC curves for the TATP vapour indicated the true positive rate of the walkthrough detection portal exceeded $99 \%$ with the false positive rate below $0.1 \%$, even when only $1 \mathrm{~mL}$ TATP vapour was tested. It is concluded from the ROC curves that this detection portal system has sufficient sensitivity and selectivity for detecting TATP in places where many people come and go.
\end{abstract}

Keywords: homeland security, improvised explosives, mass spectrometry.

\section{INTRODUCTION}

The threats of terrorism and criminal bombing have become a serious problem for all countries. It has recently become possible for the general public to easily access information on the synthesis of improvised explosives based on everyday goods via the Internet [1]. In Japan, for example, triacetone triperoxide (TATP) is one of the most worrying improvised explosives, even though military explosives and industrial explosives are strictly controlled. To maintain a safe society, it is necessary to develop technologies for detecting hidden explosives.

Two methods are primarily used to detect hidden explosives: bulk detection and trace detection. With trace detection, chemical analysis methods such as mass spectrometry and ion-mobility spectroscopy are applied to check for the existence of trace contaminants of explosives on a passenger's body, clothes, or luggage [2-8].

We have already developed explosives trace detectors (ETDs) based on mass spectrometry for improving airport security [9, 10]; however, the throughput of current ETDs is about 200 persons/hour, which is not high enough for use in busy public places. To solve the throughput problem, we developed a high-throughput walkthrough portal for detecting TATP vapour $[11,12]$. In the portal system, gasses emitted from traces of chemicals attached to the body, clothes, or luggage are collected by a push-pull vapour sampler, which consists of a blower unit and a sampling-ports unit. The air sampled by the vapour sampler is introduced 
into an ion-source unit of a mass spectrometer, and the ions produced by the ion source are analysed by the mass spectrometer. The throughput of the portal system is 1,200 persons per hour for detection (or one person every 3 seconds).

An evaluation of the false alarm rates is necessary for practical use of the portal system. We previously reported the false alarm rates evaluated from field test results in a train station environment [12]. In the field test, the walkthrough portal system was located in the event space of a train station and over 3,000 volunteers were checked using the system.

To evaluate the performance of the walkthrough portal system under a more realistic situation, we placed the system near the automated ticket gate of a train station and collected background signal data from approximately 10,000 passengers. Based on the background signal data obtained in the field test and calibration curves for the TATP vapour, we also drew receiver operating characteristic (ROC) curves for the walkthrough portal system.

This report discusses the false alarm rates of the walkthrough portal system at an automated ticket gate area of a train station and the relationship between a true positive rate and false positive rate for detecting TATP vapour.

\section{EXPERIMENTAL DETAILS}

\subsection{Walkthrough portal system}

A schematic image of the walkthrough portal system for detecting TATP vapour is shown in Fig. 1. Details of the walkthrough portal system are described in our previous reports $[11,12]$. Briefly, the system consists of a vapour sampler, an atmospheric-pressure chemical ionisation (APCI) ion source, and a linear ion-trap mass spectrometer with wire electrodes.

Sample gasses emitted from the trace contaminants of chemicals adhering to the body, clothes, or luggage are collected by a push-pull vapour sampler, which consists of an air blower and a sampling-ports unit. The temperature of the air flow supplied by the air blower

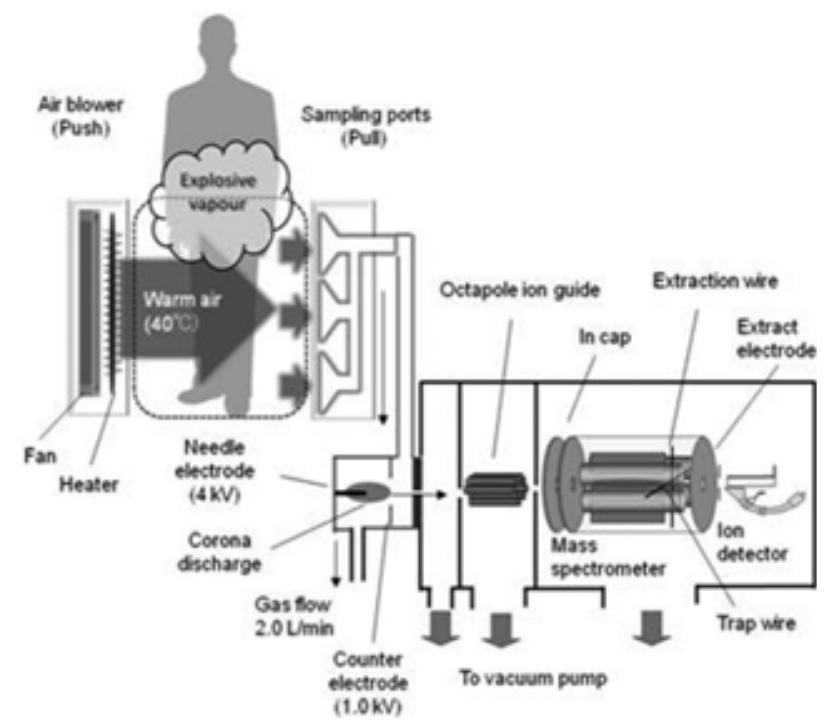

Figure 1: Schematic image of walkthrough detection portal system. 
was set at $40^{\circ} \mathrm{C}$ to vaporise the chemicals. The flow rate and speed of the air flow between the air blower and the sampling ports unit were set at $2 \mathrm{~m}^{3} / \mathrm{min}$ and $4 \mathrm{~m} / \mathrm{s}$, respectively.

The sample gas is collected by the sampling ports unit and introduced into an APCI ion source via a metal tube. The flow rate of the sampled gas was set at $2 \mathrm{~L} / \mathrm{min}$. For passenger safety, the sampling ports were not heated. The temperature of the metal tube was set at $100^{\circ} \mathrm{C}$.

The sample gas introduced into the APCI ion source is ionised by an ion-molecule reaction. A needle electrode is used to produce a corona discharge. Typical ionisation reactions in a positive APCI mode are as follows:

1. Primary ionisation by a corona discharge to produce hydronium ions $\left(\mathrm{H}_{3} \mathrm{O}^{+}\right)$

$$
\begin{aligned}
\mathrm{N}_{2}+\mathrm{e}^{-} & \rightarrow \mathrm{N}_{2}^{+}+2 \mathrm{e}^{-} \\
\mathrm{N}_{2}+\mathrm{N}_{2}^{+} & \rightarrow \mathrm{N}_{4}^{+} \\
\mathrm{H}_{2} \mathrm{O}+\mathrm{N}_{4}^{+} & \rightarrow \mathrm{H}_{2} \mathrm{O}^{+}+2 \mathrm{~N}_{2} \\
\mathrm{H}_{2} \mathrm{O}+\mathrm{H}_{2} \mathrm{O}^{+} & \rightarrow \mathrm{H}_{3} \mathrm{O}^{+}+\mathrm{OH}
\end{aligned}
$$

2. Secondary ionisation by an ion-molecule reaction to produce a protonated molecule $\left((\mathrm{M}+\mathrm{H})^{+}\right)$, where $\mathrm{M}$ indicates the sample molecule to be analysed

$$
\mathrm{M}+\mathrm{H}_{3} \mathrm{O}^{+} \rightarrow(\mathrm{M}+\mathrm{H})^{+}+\mathrm{H}_{2} \mathrm{O}
$$

We use our original APCI ion source called "counter flow introduction (CFI)" [9, 10]. In the ion source, the sample gas is introduced between a first aperture and a counter electrode, and the sample gas flows toward the corona-discharge region. The CFI-APCI ion source has several advantages. For example, it has high ionisation efficiency in negative APCI mode (which is commonly used for detecting military and industrial explosives) and achieves a long-term, continuous operation in practical applications.

High voltage $(4 \mathrm{kV})$ was applied to a needle electrode to produce the corona discharge. A voltage of $1 \mathrm{kV}$ was applied to the counter electrode to extract the ions in the direction opposite to the gas flow by an electric field. The ions were then introduced into a vacuum region via a differential pumping region. The diameter and length of the first aperture were $0.13 \mathrm{~mm}$ and $12 \mathrm{~mm}$, respectively. The pressure between the first and second aperture was $400 \mathrm{~Pa}$. A drift voltage of $10 \mathrm{~V}$ was applied between the first and second apertures. An octapole ion guide was installed between the second and third apertures to focus the ions. The voltage and frequency of the AC signal applied to the octapole were $50 \mathrm{~V}$ and $1,500 \mathrm{kHz}$, respectively.

In the vacuum region, the ions are analysed by our original mass spectrometer, a linearion-trap mass spectrometer with wire electrodes (wire-LIT) [13]. The wire-LIT consists of an inlet lens, quadrupole rods, a trap wire, an extraction wire, and an extraction lens. Two phases of a trapping AC voltage $(834 \mathrm{kHz})$ are applied to the rod pairs. The wire electrodes are placed between the quadrupole rods. The trap wire and extraction wire are aligned orthogonally to each other. The trap wire confines ions inside and the extraction wire extracts excited ions only in the axial direction. The extraction lens is aligned in parallel to the extraction wire. Helium buffer gas at 0.3 mTorr is introduced into the wire-LIT to remove the kinetic energy of the injected ions by means of collisions between the helium and ions.

The mass analysis time of the wire-LIT was set at $0.1 \mathrm{~s} / \mathrm{scan}$; therefore, roughly 10 mass spectra are accumulated in the data processor of the mass spectrometer every second.

The detection throughput of the walkthrough portal system was 1,200 persons per hour (one person every 3 seconds). 


\subsection{Setting of the walkthrough portal system for field test}

Two of the walkthrough portal systems described above were tested at Yokohama train station (with the cooperation of Tokyu Corporation and Yokohama Minatomirai Railway Company) to obtain background signals under practical field conditions and to evaluate the false positive rate for TATP vapour detection. One of the systems was located at the main exit area, with the other located at the south exit area of the station.

Photographs of the field test taken at the main exit area of the train station are shown in Fig. 2. The detection portal system was set near an automated ticked gate, as shown in Fig. 2(a). The air blower, sampling ports, and mass spectrometer were set at the exit side of the automated ticket gate, as shown in Fig. 2(b). Another system was set at the south exit area in the same way as described above.

Passengers who passed through the automated ticket gate were checked by the walkthrough portal systems. In this field test, signal intensities at $\mathrm{m} / \mathrm{z} 75,77$ and 91 were monitored to detect TATP vapour.

The field test was conducted on 12 through 14 November 2010 from 9 AM to 5 PM and 9,951 passengers were checked by the walkthrough portal systems.

\subsection{Detection procedures of the field test}

The data processing procedures that determine whether TATP vapours are detected in the field test are listed below:

1. Background signal data are accumulated by the mass spectrometer.

2. Average and standard deviations $(\sigma)$ of the background signals for each $\mathrm{m} / \mathrm{z}$ are calculated by a data processor and the alarm levels are set at [average background signal $+3 \sigma$ ] for each $m / z$. The background signal always changes for various reasons. For example, a change in humidity in the ambient air will affect the ionisation efficiency of the APCI ion source. To avoid this problem, we developed an alarm level control program to adjust the alarm levels in real time (hereafter, referred to as the "automated alarm level control").

3. The walkthrough portal systems utilise optical sensors to determine the time when a passenger passes through the system. Mass spectra obtained within 5 seconds after a passenger passes through the walkthrough portal system, roughly 50 mass spectra per
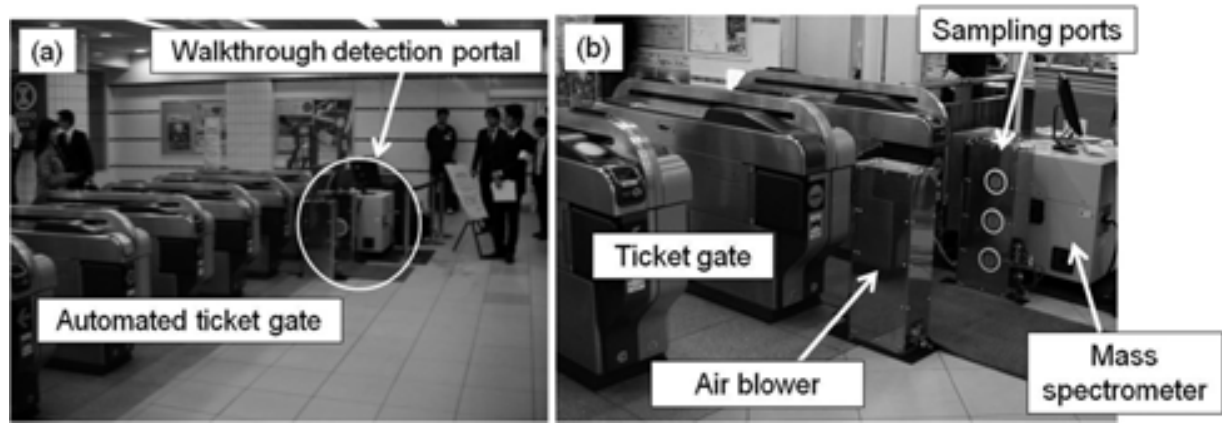

Figure 2: Photographs of (a) field test area at Yokohama train station, and (b) setup of the air blower, sampling ports, and mass spectrometer. 
passenger, are analysed. Due to statistical characteristics of the background signals, the obtained signals will frequently exceed the predetermined alarm levels (average background signal $+3 \sigma$ ) for each $\mathrm{m} / \mathrm{z}$. To avoid false positive alarms based on random noise, we define an "effective signal", which refers to the case in which two signals continuously exceed the alarm level.

\subsection{Sample}

Sample powders of TATP were synthesised in our laboratory.

\section{RESULTS AND DISCUSSION}

\subsection{Field test results}

In our previous reports, two strong signals at $\mathrm{m} / \mathrm{z} 75$ and 77 were chosen as the detection markers of TATP vapour [11, 12]; however, by optimising the operating parameters of the wire-LIT, the $m / z 91$ signal became dominant. Figure 3 shows (a) the molecular structure of TATP and (b) the mass spectrum of TATP vapour obtained by the positive APCI mode. Based on the molecular structure of TATP, it is assumed that the $m / z 91$ signal would be $\left(\mathrm{C}_{3} \mathrm{H}_{6} \mathrm{O}_{3}+\mathrm{H}\right)^{+}$ [14]; therefore, we used the $m / z, 75,77$ and 91 signals as detection markers for TATP in this field test.

Table 1 summarises the detection results for TATP vapour measured at the train station. As shown in this table, the signals for $m / z, 75,77$, and 91 frequently exceeded the $3 \sigma$ alarm level. The preliminary walkthrough detection results show that when a TATP sample was on a subject, the TATP vapour was detected at least twice, because the data sampling period $(0.5 \mathrm{~s}$ in the previous experiment, although the sampling period was set at $0.1 \mathrm{~s}$ in this field test) was short enough to detect plural signals [11, 12]. It was, therefore, assumed that almost all the signals that exceeded the $3 \sigma$ alarm level were random fluctuations of the background signal. Accordingly, an "effective signal" is defined as one that continuously exceeds the $3 \sigma$ alarm level. The numbers of effective signals for each $\mathrm{m} / \mathrm{z}$ are also listed in Table 1 . The false positive rates for the 9,951 passengers estimated from the numbers of effective signals were $0.50 \%, 1.97 \%$, and $0.49 \%$ for $m / z 75,77$, and 91 , respectively.

To reduce the false positive rate, we evaluated the use of multi-marker detection logic for TATP [12]. TATP vapour gives three signals on the mass spectrum, as shown in Fig. 3(b).

(a)
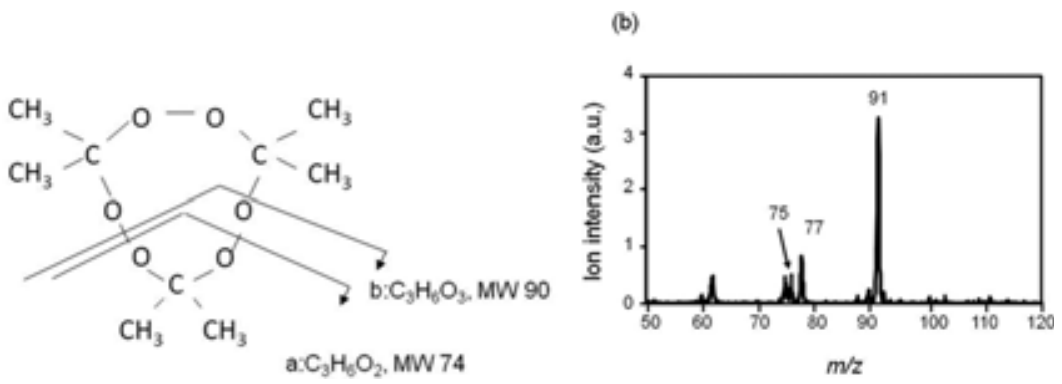

Figure 3: (a) Molecular structure of TATP, and (b) typical mass spectrum of TATP vapour in positive APCI mode. 
We checked the case in which the two or three markers for TATP were obtained as effective signals at the same time. During the field test, $\mathrm{m} / \mathrm{z} 77$ and 91 gave effective signals at the same time, but the false-positive rate was $0.01 \%$, as indicated in Table 1. It is, therefore, concluded that the multi-marker detection logic for TATP effectively reduced the false positive alarms for practical field operation of the detection portal system.

3.2 Calibration curves for TATP vapour obtained by the walkthrough detection portal system

Unfortunately, we were not able to use actual explosive TATP at the train station. Instead, we obtained calibration curves of the walkthrough portal system for TATP vapour in our laboratory. We sampled the headspace vapour of a TATP sample bottle at room temperature using a syringe. This sampled vapour was then injected between the air blower and the sampling ports. The TATP signals were obtained every 2 seconds after the sample vapour was injected into the sampling space. The obtained calibration curves for $m / z, 75,77$, and 91 are shown in Fig. 4. As shown in this figure, the $1 \mathrm{~mL}$ of TATP vapour, roughly $0.6 \mu \mathrm{g}$ in weight, was enough to be detected by the walkthrough detection portal system.

\subsection{ROC curves}

An ROC curve shows the relationship between a true positive rate and a false positive rate. Typical signal distributions and the ROC curve are shown in Fig. 5(a) and (b), respectively. In general, the distributions of the background and the signal overlap, as shown in Fig. 5(a). If the alarm level that determines TATP vapour detection is set at a very low intensity, false positive alarms will occur frequently, even though all signals will be detected. If the alarm level is set at a very high intensity, on the other hand, false positive alarms will not occur, but we will lose part of the signals. The ROC curve is obtained by changing the alarm level from low intensity to high intensity in the distributions shown in Fig. 5(a), and plotting the relationship between the true positive rate and the false positive rate, as shown in Fig. 5(b).

To obtain suitable ROC curves for the walkthrough detection portal, it is necessary to conduct detection tests using real explosives in practical field environments; however, it is strictly

Table 1: Number of signals exceeding the alarm level, number of effective signals, and false positive rate for each $\mathrm{m} / \mathrm{z}$. Effective signal means the case in which two signals continuously exceeded $3 \sigma$ for each $\mathrm{m} / \mathrm{z}$.

\begin{tabular}{lccc}
\hline $\begin{array}{l}\text { Detection marker } \\
(\mathrm{m} / \mathrm{z})\end{array}$ & $\begin{array}{c}\text { Signal over } 3 \sigma \\
\text { (number of times) }\end{array}$ & $\begin{array}{c}\text { Effective signal } \\
\text { (number of times) }\end{array}$ & $\begin{array}{c}\text { False-positive rate for } \\
9,951 \text { passengers }(\%)\end{array}$ \\
\hline 75 & 3,859 & 50 & 0.50 \\
77 & 8,462 & 196 & 1.97 \\
91 & 2,183 & 49 & 0.49 \\
75 and 77 & - & 0 & 0.00 \\
77 and 91 & - & 1 & 0.01 \\
75 and 91 & - & 0 & 0.00 \\
75,77 , and 91 & - & 0 & 0.00 \\
\hline
\end{tabular}


(a)

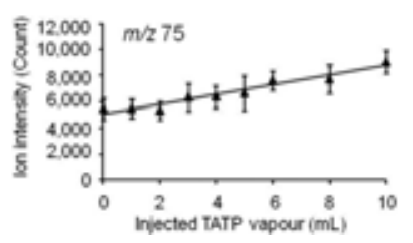

(b)

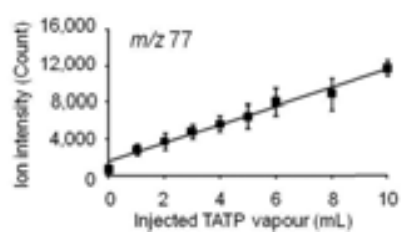

(c)

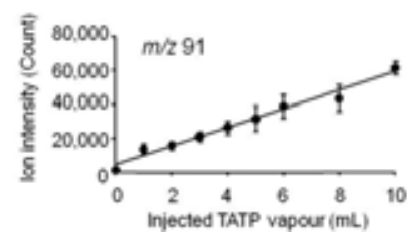

Figure 4: Calibration curves of walkthrough portal system for TATP vapour obtained at our laboratory. The monitored $\mathrm{m} / \mathrm{z}$ was (a) 75, (b) 77, and (c) 91 .

(a)

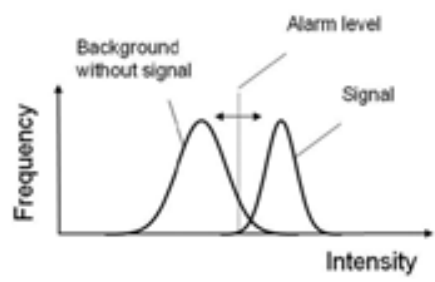

(b)

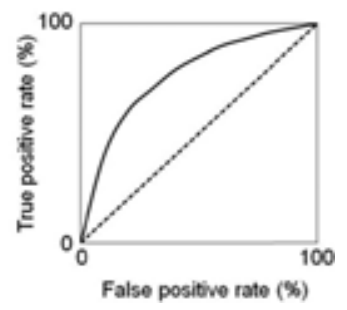

Figure 5: Example of receiver operating characteristic (ROC) curve. (a) General distribution of the background signal and the signal obtained from a sample, and (b) the typical ROC curve.

(a) $m / 277$

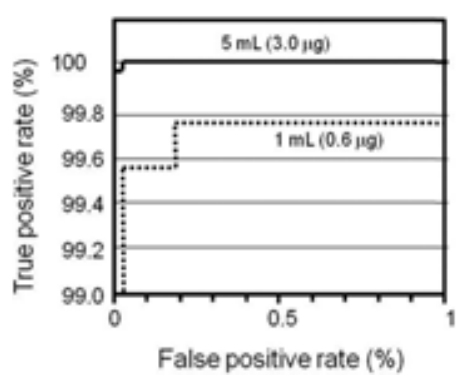

(b) $m / 291$

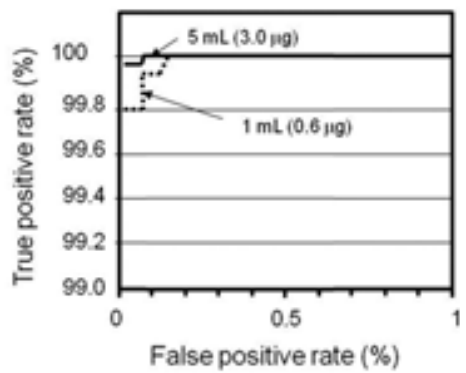

Figure 6: ROC curves of walkthrough portal system for different TATP vapour concentrations. The monitored $m / z$ was (a) 77 and (b) 91 . Samples of headspace vapour in the TATP bottle were taken with a syringe and the sampled gases were injected between the air blower and sampling ports. The volume of injected sample gases were $1 \mathrm{~mL}$ (broken line) and $5 \mathrm{~mL}$ (solid line), corresponding roughly to $0.6 \mu \mathrm{g}$ and $3 \mu \mathrm{g}$ in TATP, respectively.

forbidden to bring explosive materials into train stations in Japan, even for scientific research. To mitigate this problem, we simulated the ROC curves of the walkthrough portal system for detecting TATP vapour by using all background signal data accumulated at the field test and the calibration curves shown in Fig. 4. Figure 6 plots the ROC curves of the walkthrough detection portal system for $m / z$ (a) 77 and (b) 91, respectively. As shown in Fig. 6, the true positive rate of 
the walkthrough detection portal exceeded $99 \%$ with the false positive rate below $0.1 \%$ for both detection markers, $m / z, 77$ and 91 , by choosing the appropriate alarm levels.

\section{CONCLUSION}

False alarm rates of a walkthrough detection portal system were evaluated by using the results of field test conducted at Yokohama train station. By using both the automated alarm level control program and the multi-marker detection logic, we obtained the estimated false positive rate of the walkthrough portal system for TATP detection below $0.01 \%$. Furthermore, from the ROC curves obtained in the field test results and the calibration curves of the portal system for the TATP vapour, the true positive rate of the walkthrough detection portal exceeded $99 \%$ with the false positive rate below $0.1 \%$, even when testing only $1 \mathrm{~mL}$ of TATP vapour.

We, therefore, concluded that the portal system was able to detect the TATP vapour in the train station environment with sufficient sensitivity and selectivity. The walkthrough portal system will be useful in preventing terrorist attacks or criminal bombings that use TATP in crowded public places.

Our next challenge will be to expand the detection target of the high throughput explosives trace detection system from volatile compounds, such as TATP and inflammable liquids, to nonvolatile explosives, such as plastic explosives. We will also work on developing a compact mass spectrometer to install the whole system, which consists of a sampling unit, ion source, mass spectrometer, and data processor, into a security gate.

\section{ACKNOWLEDGMENTS}

This work was supported by the Ministry of Education, Culture, Sports, Science and Technology (MEXT), Japan. We also thank the Ministry of Land, Infrastructure, Transport and Tourism, the National Police Agency, Tokyu Corporation, and Yokohama Minatomirai Railway Company for their support in carrying out the field test at Yokohama train station.

\section{REFERENCES}

[1] Schubert, H. \& Kuzentsov, A., Detection and Disposal of Improvised Explosives, Springer: Dordrecht, The Netherlands, 2006. doi: http://dx.doi.org/10.1007/978-14020-4887-6

[2] Yinon, J., Mass spectrometry of explosives: nitro compounds, nitrate esters, and nitramines. Mass Spectrometry. Reviews 1, p. 257, 1982.

[3] McLuckey, S.A., Glish, G.L. \& Asano, K.G., Coupling of an atmospheric-sampling ion source with an ion-trap mass spectrometer. Anaytical. Chimica. Acta 225, p. 25, 1989.

[4] Yinon, J. \& Zitrin, S., Modern Methods and Applications in Analysis of Explosives, John Wiley and Sons Ltd: New York, 1993.

[5] McLuckey, S.A., Goeringer, D.E., Asano, K.G., Vaidyanathan, G. \& Stephenson Jr., J.L., High explosives vapor detection by glow discharge-ion trap mass spectrometry. Rapid Communications. in Mass Spectrometry 10, p. 287, 1996. http://dx.doi.org/10.1002/ (SICI)1097-0231(199602)10:3<287::AID-RCM429>3.0.CO;2-H

[6] Evans, C.S., Sleeman, R., Luke, J. \& Keely, B.J., A rapid and efficient mass spectrometric method for the analysis of explosives. Rapid Communications. in Mass Spectrometry 16, p. 1883, 2002. doi: http://dx.doi.org/10.1002/rcm.799

[7] Crowson, A. \& Beardah, M.S., Development of an LC/MS method for the trace analysis of hexamethylenetriperoxidediamine (HMTD). Analyst 126, p. 1698, 2001.

[8] Xu, X., van de Craats, A.M., Kok, E.M. \& de Bruyn, P.C.A.M., Trace analysis of peroxide explosives by high performance liquid chromatography-atmospheric pressure 
chemical ionization-tandem mass spectrometry (HPLC-APCI-MS/MS) for forensic applications. Journal. Forensic Science 49, p. 1, 2004.

[9] Kojima, K., Sakairi, M., Takada, Y. \& Nakamura, J., Vapor detection of TNT and RDX using atmospheric pressure chemical ionization mass spectrometry with counter-flow introduction (CFI). Journal. Mass Spectrometry. Society of Japan 48, p. 360, 2000.

[10] Takada, Y., Nagano, H., Suga, M., Hashimoto, Y., Yamada, M., Sakairi, M., Kusumoto, K., Ota, T. \& Nakamura, J., Detection of military explosives by atmospheric pressure chemical ionization mass spectrometry with counter flow introduction. Propellants, Explosives, Pyrotechnics 27, p. 224, 2002. doi: http://dx.doi.org/10.1002/15214087(200209)27:4<224::AID-PREP224>3.0.CO;2-V

[11] Takada, Y., Nagano, H., Suzuki, Y., Sugiyama, M., Nakajima, E., Hashimoto, Y. \& Sakairi, M., High-throughput walkthrough detection portal for counter terrorism: detection of triacetone triperoxide (TATP) vapor by atmospheric-pressure chemical ionization ion trap mass spectrometry. Rapid Communications. in Mass Spectrometry 25, p. 2448, 2011.

[12] Takada, Y., Suzuki, Y., Nagano, H., Sugiyama, M., Nakajima, E., Sugaya, M., Hashimoto, Y. \& Sakairi, M., High-Throughput Walkthrough Detection Portal as a Measure for Counter Terrorism: Design of a Vapor Sampler for Detecting Triacetone Triperoxide Vapor by Atmospheric-Pressure Chemical-Ionization Ion-Trap Mass Spectrometry. IEEE Sensors Journal, in press.

[13] Sugiyama, M., Hasegawa, H. \& Hashimoto, Y., Mass-selective axial ejection from a linear ion trap with a direct current extraction field. Rapid Communications. in Mass Spectrometry 23, p. 2917, 2009.

[14] Sigman, M.E., Clark, C.D., Fidler, R., Geiger, C.L. \& Clausen, C.A., Analysis of triacetone triperoxide by gas chromatography/mass spectrometry and gas chromatography/ tandem mass spectrometry by electron and chemical ionization. Rapid Communications. in Mass Spectrometry 20, p. 2851, 2006. 